\title{
Effects of resonant interface states on tunneling magnetoresistance
}

\author{
O. Wunnicke, N. Papanikolaou, R. Zeller, and P. H. Dederichs \\ Institut für Festkörperforschung, Forschungszentrum Jülich, D-52425 Jülich, Germany \\ V. Drchal and J. Kudrnovský \\ Institute of Physics, Academy of Sciences of the Czech Republic, CZ-18040 Praha 8, Czech Republic
}

(Received 2 October 2001; published 22 January 2002)

\begin{abstract}
Based on model and ab initio calculations we discuss the effect of resonant interface states on the conductance of epitaxial tunnel junctions. In particular we show that the "hot spots" found by several groups in $a b$ initio calculations of symmetrical barriers of the $\mathbf{k}_{\|}$-resolved conductance can be explained by the formation of bonding and antibonding hybrids between the interface states on both sides of the barrier. If the resonance condition for these hybrid states is met, the electron tunnels through the barrier without attenuation. Even when both hybrid states move together and form a single resonance, strongly enhanced transmission is still observed. The effect explains why, for intermediate barrier thicknesses, the tunneling conductance can be dominated by interface states, although hot spots only occur in a tiny fraction of the surface Brillouin zone.
\end{abstract}

DOI: 10.1103/PhysRevB.65.064425

PACS number(s): 72.25.-b, 73.20.-r, 73.40.Rw, 73.40.Gk

The tunneling magnetoresistance (TMR) of magnetic tunnel junctions consisting of ferromagnet|insulator|ferromagnet layers has attracted a strong scientific interest, partly due to their potential application as magnetic random access memories. Miyazaki and Tezuka ${ }^{1}$ and Moodera et al. ${ }^{2}$ were able to obtain TMR ratios up to $20 \%$ in room-temperature experiments and recently room-temperature values of more than $50 \%$ were reported by various groups. The understanding of the TMR and of the electronic structure has not progressed equally quickly. Model calculations ${ }^{3,4}$ have shed light on various aspects of the effect, but only recently have $a b$ initio calculations of the electronic structure and the spindependent transport been reported..$^{5-8}$

In this paper, we will consider the tunneling through epitaxial junctions, which are characterized by two-dimensional periodicity. Here recent $a b$ initio calculations of the $\mathbf{k}_{\|}$-resolved conductance show a very interesting phenomenon: for certain discrete $\mathbf{k}_{\|}$values "hot spots" or "spikes" appear in the transmitted intensity, showing that electrons with such $\mathbf{k}_{\|}$values can apparently tunnel through the junction with no or very little attenuation while all other states are very strongly damped..$^{9-11}$ This effect occurs only in the minority band of the ferromagnet and only for ferromagnetic coupling. If present, it can dominate the tunnel characteristics for intermediate thicknesses. For large thicknesses, in the asymptotic limit, the behavior is determined by the complex band structure of the insulator, ${ }^{12}$ i.e., by those metal-inducedgap states, which have the smallest imaginary part of the perpendicular component $k_{z}$ of the Bloch vector. An example for such hot spots is given in Fig. 1, showing the results of $a b$ initio Korringa-Kohn-Rostoker calculations for a junction consisting of two fcc $\mathrm{Co}(001)$ half-crystals separated by 4 monolayers (ML) of vacuum. The results are based on density-functional theory in the local-density approximation and the Landauer formula for the conductance. We have chosen a vacuum layer as the simplest model of an insulating barrier. Quite similar effects are also found in the calculations $^{9-11}$ for insulating barriers.
The $\mathbf{k}_{\|}$-resolved conductance is plotted in the twodimensional (001) Brillouin zone at the Fermi level, for the case of ferromagnetic-moment alignment of the two Co half crystals in Fig. 1(a) for the majority electrons and in Fig. 1(b) for the minority ones. Figure 1(c) gives the conductance for the case of antiparallel alignment. The majority conductance shows a smooth peak at the $\bar{\Gamma}$ point. This is the "normal" behavior expected for a potential barrier, since electrons with perpendicular incidence experience the smallest decay in the vacuum region. As explained in terms of the complex band structure, ${ }^{12}$ this behavior is also expected for most insulators. In contrast to this the minority conductance is dominated by four double peaks in the $\bar{\Gamma}-\bar{X}$ directions with extremely high intensity, compared to which the contributions from other peaks seem to be negligible. The structure of these double peaks will be discussed later. For the case of antiparallel alignment [Fig. 1(c)] the $\mathbf{k}_{\|}$-resolved conductance shows both features, i.e., a smooth peak at the $\bar{\Gamma}$ point with similar intensity as the majority conductance [Fig. 1(a)] and four double peaks at the same $\mathbf{k}_{\|}$values in the $\bar{\Gamma}-\bar{X}$ directions. However, compared to the minority case [Fig. $1(b)]$, the intensity of these hot spots is reduced by more than two orders of magnitude so that they are of minor importance. While it is tempting to attribute the hot spots to numerical problems in the complicated evaluation of the conductance, several groups have demonstrated recently that these spots are connected with the occurrence of interface states in the minority band..$^{9-11}$ Yet details of the mechanism, in particular, why these hot spots are practically not damped, are still not properly understood, ${ }^{13}$ as we believe.

Here we present a detailed investigation of the properties and the occurrence of such hot spots. As we will show, these spectacular spikes only occur for symmetrical or nearly symmetrical barriers and are due to the formation of bonding and antibonding hybrids of the interface states on both sides of the barrier. As long as the bonding-antibonding splitting of these two hybrids is larger than the genuine width of the interface resonance the particle can tunnel through the barrier 
(a)

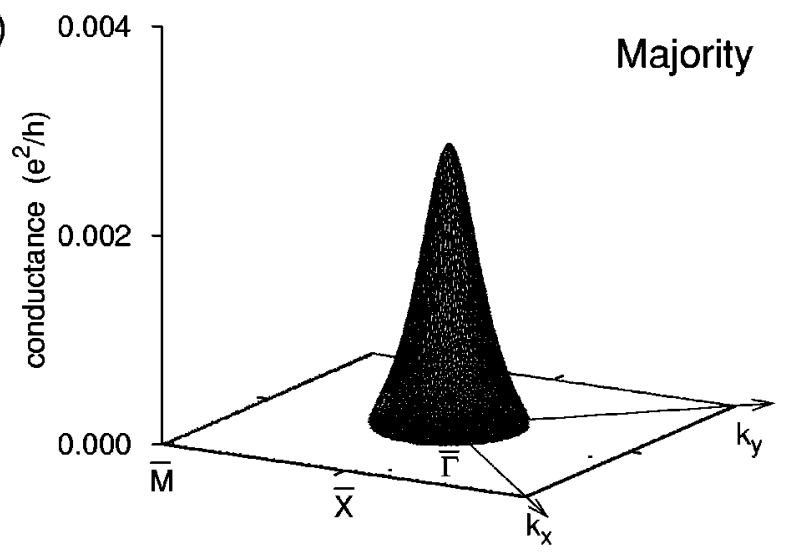

(b)

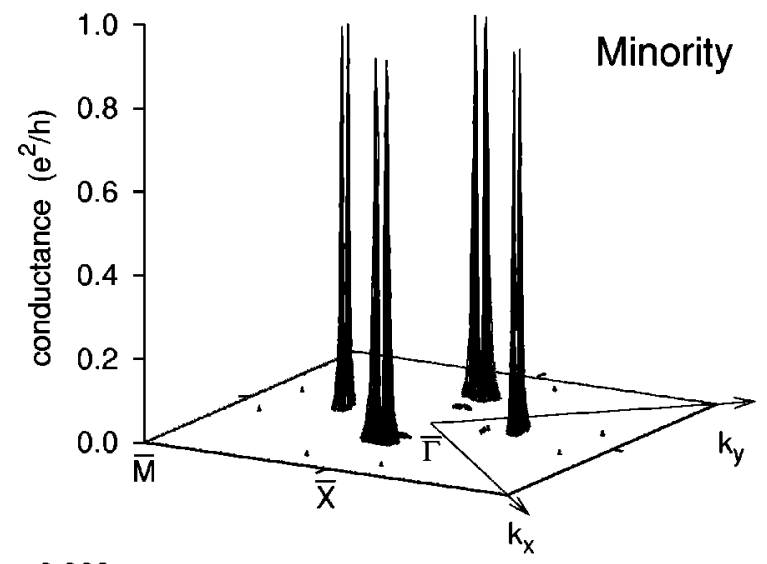

(c)

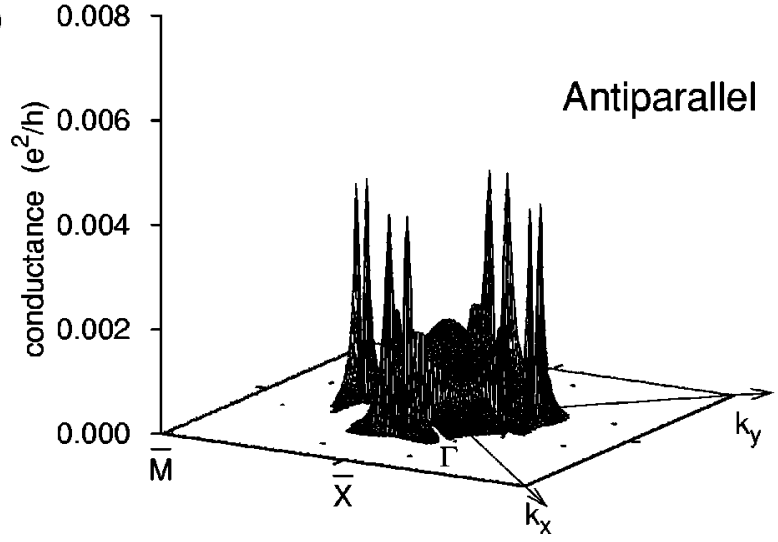

FIG. 1. $\mathbf{k}_{\|}$-resolved $a b$ initio conductance plot in the SBZ for a $\mathrm{Co}|4 \mathrm{vac}| \mathrm{Co}$ tunnel junction. The figure shows the conductance for parallel aligned moments in the majority band (a) and the minority band (b) as well as the conductance for antiparallel aligned moments (c) of the Co half crystals.

without attenuation, i.e., the barrier becomes fully transparent. On the other hand for larger thicknesses both hybrid levels fuse together into a single resonance, full transmission is no longer possible, and attenuation sets in. However the hybridization effect is still important and the peaks dominate the behavior for an intermediate thickness region, before for even large thicknesses the normal resonance behavior as in Fig. 1(c) for the antiparallel alignment is obtained. We will discuss this effect by two methods, first by a simple analytical model and second by $a b$ initio calculations for the
Co|vacuum|Co junction using the tight-binding (TB) linear muffin-tin orbital (TBLMTO) method.

In the model calculation we assume a barrier with a constant potential of height $V_{B}$ and thickness $D$. The potential $V(x, y, z)$ in the two half crystals is independent of $z$, the direction normal to the barrier. For the in-plane $x$ and $y$ dependencies we assume a weak potential corrugation in the $x$ direction described by, e.g., the Fourier coefficients $V_{G_{x}}$ and $V_{-G_{x}}$ for the smallest reciprocal-lattice vectors in the $x$ direction. The eigenfunctions are then calculated in the nearlyfree-electron approximation. In the following we discuss only the case of perpendicular incidence $\mathbf{k}_{\|}=0$. In the unperturbed case, i.e., $V_{G_{x}}=0$, we obtain an incident wave $e^{i k_{z} z}$ with energy $E=k_{z}^{2}$ and a second wave $e^{i k_{z} z} \cos \left(G_{x} x\right)$ with energy $E=k_{z}^{2}+G_{x}^{2}$. For $V_{G_{x}} \neq 0$, but small, the new eigenfunctions, i.e., the Bloch waves, still have the same dominating plane-wave character, either $e^{i k_{z} z}$ or $e^{i k_{z} z} \cos \left(G_{x} x\right)$, but they also have a small admixture of the second plane-wave component, so that the energy dispersion is only slightly changed. In addition we introduce two attractive $\delta$ functions $-\beta_{1} \delta(z+D / 2)$ and $-\beta_{2} \delta(z-D / 2)$ at the interfaces on both sides of the barrier, which allow us to introduce interface states. The solutions in the two half crystals and in the barrier are matched at the interfaces $z=-D / 2$ and $z=+D / 2$. If the $\beta_{1}$ (or $\beta_{2}$ ) value is sufficiently large, it introduces at the left (or right) interface an interface state for an energy below the bottom of each band. The interface state below the lower band $\left(k_{z}^{2}\right)$ is localized, while the one below the higher band $\left(k_{z}^{2}+G_{x}^{2}\right)$ is resonant, since it hybridizes weakly with the Bloch waves of the lower band $\left(k_{z}^{2}\right)$.

We consider now a Bloch wave $\mathbf{k}=\left(0,0, k_{z}\right)$ incident on the barrier. If the barrier is sufficiently high, the transition probability $|t|^{2}$ is relatively small, as is shown in Fig. 2(a) for energies away from the energy of the resonant interface states.

In the vicinity of the resonance, we observe anomalies. The dashed curve gives the results for a single resonance on one side of the interface, obtained by allowing only $\beta_{1}$ or $\beta_{2}$ to be nonzero. At the resonance energy we obtain then a maximum in the transmission, thus an enhanced tunneling probability which is due to the enhancement of the incoming wave function by the large amplitude of the resonant interface state. The following zero of $|t|^{2}$, an antiresonance, can be explained by the Fano effect. ${ }^{14}$ While this behavior is as expected, a dramatic effect occurs in the case of two degenerate interface states on both sides of the barrier, created by two equal $\delta$ potentials $\beta_{1}=\beta_{2}$. We observe two resonance peaks, both showing full transmission, $|t|^{2}=1$, of the incident wave. The peaks are symmetrically situated at lower and higher energies compared with the energy of the single resonance, and both are accompanied by an antiresonance. The calculation of the wave functions [Fig. 2(b)] shows that for the lower peak both interface states form a bonding hybrid (solid line) and for the higher peak an antibonding hybrid (dashed line). Thus, for these resonances the total wave function is nearly symmetrical or antisymmetrical with respect to the center of the barrier, which directly explains the 

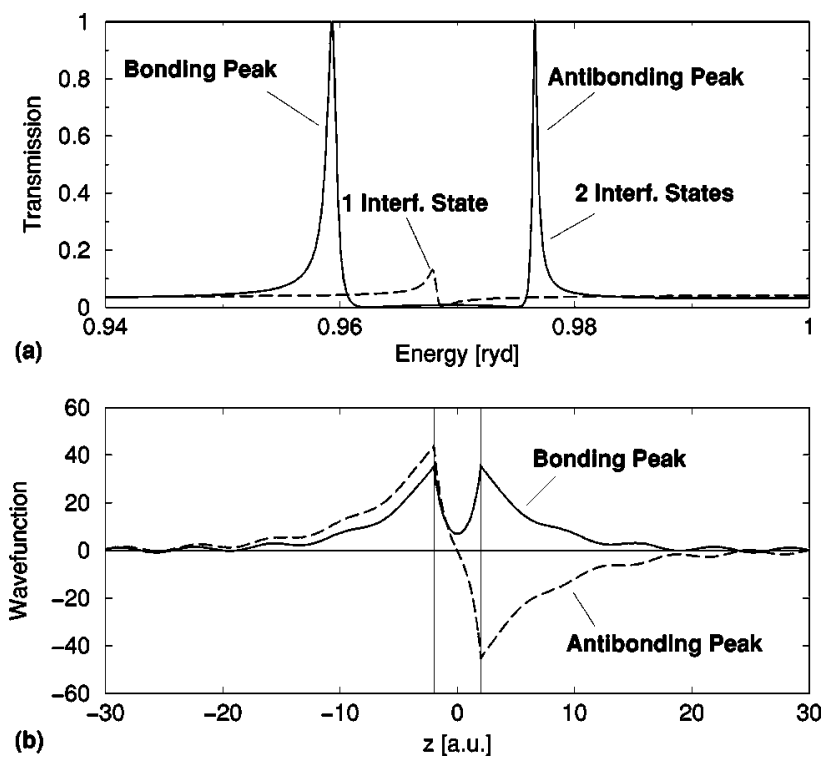

FIG. 2. Results of the analytical model. In plot (a) the transmission $|t|^{2}$ for a 4 a.u. thick barrier with one interface state (dashed line) and for a symmetrical barrier with two interface states (solid line) is shown. In plot (b) the real parts of the wave functions at the bonding (solid line) and antibonding (dashed line) peaks are plotted over the distance perpendicular to the interface plane ( $z$ axes). The interface planes at $z= \pm 2$ a.u. are indicated by two thin lines.

full transmission without any attenuation since by entering these states the particle comes to the other side of the barrier without tunneling. Note that the total wave function is not fully symmetrical or antisymmetrical, since the incident wave, incident from the left or right, breaks the symmetry of the system. Thus if the bonding-antibonding splitting $\Delta$ is larger than the natural resonance width $\Gamma, \Delta \geqslant \Gamma$, full transmission will occur, while when both resonances fuse together, $\Delta \ll \Gamma$, the normal resonance behavior is observed. This can also be understood in a time-dependent picture. If the lifetime $t_{R}=\hbar / \Gamma$ of the resonance is much larger than the hopping time $t_{H}=\hbar \pi / \Delta$, which the particle needs to coherently hop between the interface states on both sides of the barrier, then during the lifetime of the resonance, bonding and antibonding states can be formed, allowing the particle to fully penetrate the barrier via these hybrids. If $t_{R} \ll t_{H}$ or $\Delta \ll \Gamma$ this channel is no longer open and the incident wave is attenuated.

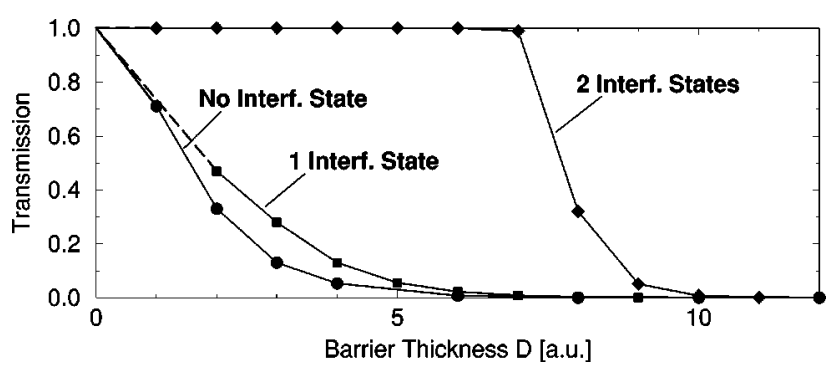

FIG. 3. Thickness dependence of the maximum transmission $|t|^{2}$ at the resonance energies for no, one, and two interface states is shown.
This effect shows up equally dramatically in Fig. 3, where the transmission probability $|t|^{2}$ at the resonance peak is plotted versus the barrier thickness $D$. In the case of no interface states $\left(\beta_{1}=\beta_{2}=0\right)$ the normal exponential dependence on the barrier thickness is obtained. For a single interface state on one side of the barrier, the tunneling is somewhat enhanced, but basically the same attenuation is observed. On the other hand, for the symmetrical barrier with degenerate interface states, full transmission is obtained for small and intermediate thicknesses and the exponential decay is strongly delayed. For very large thicknesses one finds in all three cases the same exponential decrease, i.e., with the same decay length, but with somewhat different amplitudes, being largest in the two-resonance case.

More details about the tunneling behavior can be understood from a phase-shift analysis. In the one-dimensional case, as well as in the pseudo-one-dimensional case considered here (i.e., for a given $\mathbf{k}_{\|}$component), the scattering can be described by two phase shifts $\delta_{S}(E)$ and $\delta_{A}(E)$. Here $\delta_{S}$ (or $\delta_{A}$, respectively) refers to the phase shift, when two waves with equal amplitudes (or opposite amplitudes) are incident from the left and right sides on a symmetrical barrier, so that the total wave function is symmetrical (or antisymmetrical) with respect to reflection around the center of the barrier. With these phase shifts the transmission coefficient $t(E)$ can be written as ${ }^{15,16} t=\cos \left(\delta_{S}-\delta_{A}\right) e^{i\left(\delta_{S}+\delta_{A}\right)}$. In the off-resonance energy region, the difference $\delta_{S}-\delta_{A}$ is slightly larger than $-\pi / 2(\bmod 2 \pi)$, so that the transmission $|t|^{2} \ll 1$. Near the bonding resonance, the phase shift $\delta_{S}(E)$ quickly increases by $\pi$, so that $\delta_{S}-\delta_{A}$ first crosses the resonance value $0(\bmod 2 \pi)$ with $|t|^{2}=1$, and then the value $\pi / 2$, for which $|t|^{2}=0$ (antiresonance). In the vicinity of the antibonding resonance, $\delta_{A}(E)$ increases equally quickly by $\pi$, so that the difference $\delta_{S}-\delta_{A}$ first crosses the value $\pi / 2$ for the antiresonance and then the resonance value 0 of the antibonding resonance. This is exactly the behavior of the transmission probability seen in Fig. 2(a). When the bonding and antibonding peaks move together, the "jumps" of the phase shifts $\delta_{S}(E)$ and $\delta_{A}(E)$ start to overlap and partially compensate each other in the difference $\delta_{S}-\delta_{A}$. Therefore first one loses the crossing of the value $\pi / 2$, i.e., the antiresonances disappear, while the curve $\delta_{S}-\delta_{A}$ still crosses the value 0 twice, so that the bonding and antibonding resonances still show full transmission with $|t|^{2}=1$. However, when the two resonances further move together, then the maximum of the curve $\delta_{S}(E)-\delta_{A}(E)$ becomes smaller than 0 , and at this point attenuation sets in. This discussion explains the behavior seen in Fig. 3, showing that for the symmetrical barrier one obtains at the resonance energies full transmission up to a critical thickness.

The transition region of the two-interface-state case in Fig. 3, where the transmission $|t|^{2}$ is no longer 1 , but still much larger than in the two other cases, is particularly interesting and important for understanding the conductance results of Fig. 1. In this region we have $\Delta \lesssim \Gamma$, so that the splitting $\Delta$ is smaller than the resonance width $\Gamma$ and the bonding and antibonding peaks have moved together already exhibiting a single resonance peak. Nevertheless also in this case the hybridization effect is still important. This is illus- 

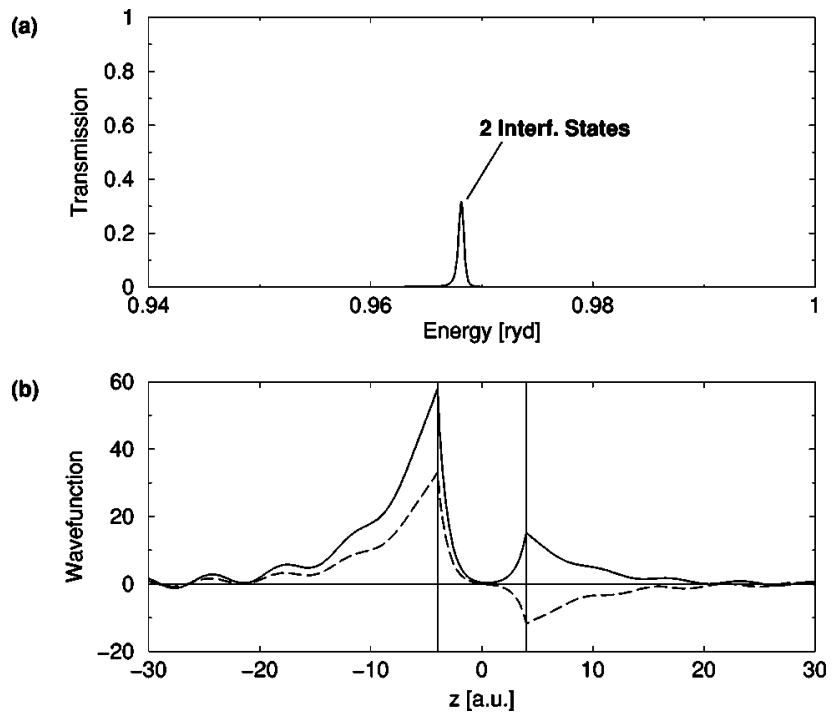

FIG. 4. Results of the analytical model. In plot (a) the transmission $|t|^{2}$ for a 8 a.u. barrier for a symmetrical barrier with two interface states is shown. In plot (b) the real part (solid line) and the imaginary part (dashed line) of the wave function at the resonance energy are plotted over the distance perpendicular of the interface plane ( $z$ axes). The interface planes at $z= \pm 4$ a.u. are indicated by two thin lines.

trated in Fig. 4 for a symmetrical barrier with 8 a.u. thickness and two interface states. For the energy at the maximum of the transmission curve the wave function [Fig. 4(b)] is clearly asymmetrical, with the real part (solid line) being bondinglike and the imaginary part (dashed line) antibondinglike. This can be explained by the fact that for $t_{R}$ $\lesssim t_{H}$, when the resonance lifetime is shorter than the hopping time, a certain percentage of electrons can still hop during the lifetime of the resonance to the interface on the other side, in this way avoiding the tunneling and enhancing the transmission probability. The importance of this effect cannot be seen well in Fig. 3, since in this thickness region all three transmissions are small, so that important differences cannot be observed, but show up, e.g., in a semilogarithmic plot.

In the following we come back to the conductances of the Co|vacuum|Co (001) barrier shown in Fig. 1 and present a detailed study of the resonance effects with very high $\mathbf{k}_{\|}$ resolution. Contrary to the previous model case in the transport calculation we vary the $\mathbf{k}_{\|}$vector in the surface Brillouin zone (SBZ) and fix the energy at the Fermi level $E_{F}$. We have calculated the transmission $T\left(\mathbf{k}_{\|}, E_{F}\right)$ by the Landauer formalism using the $a b$ initio TB-LMTO method, for which we refer to Ref. 17. To resolve the spiky structures of the hot spots a very large number (980 700) of $\mathbf{k}_{\|}$points in the irreducible part of the SBZ has been used. For the following results two fcc Co (001) half crystals have been considered, being separated by three layers of vacuum $(3 \mathrm{vac})$. For all $\mathbf{k}_{\|}$ in the SBZ, all eigenstates, either delocalized or localized, have been evaluated and analyzed by the transfer-matrix method (for a recent application to multilayers, see Ref. 18). Here we consider both the parallel alignment of the Co mo-
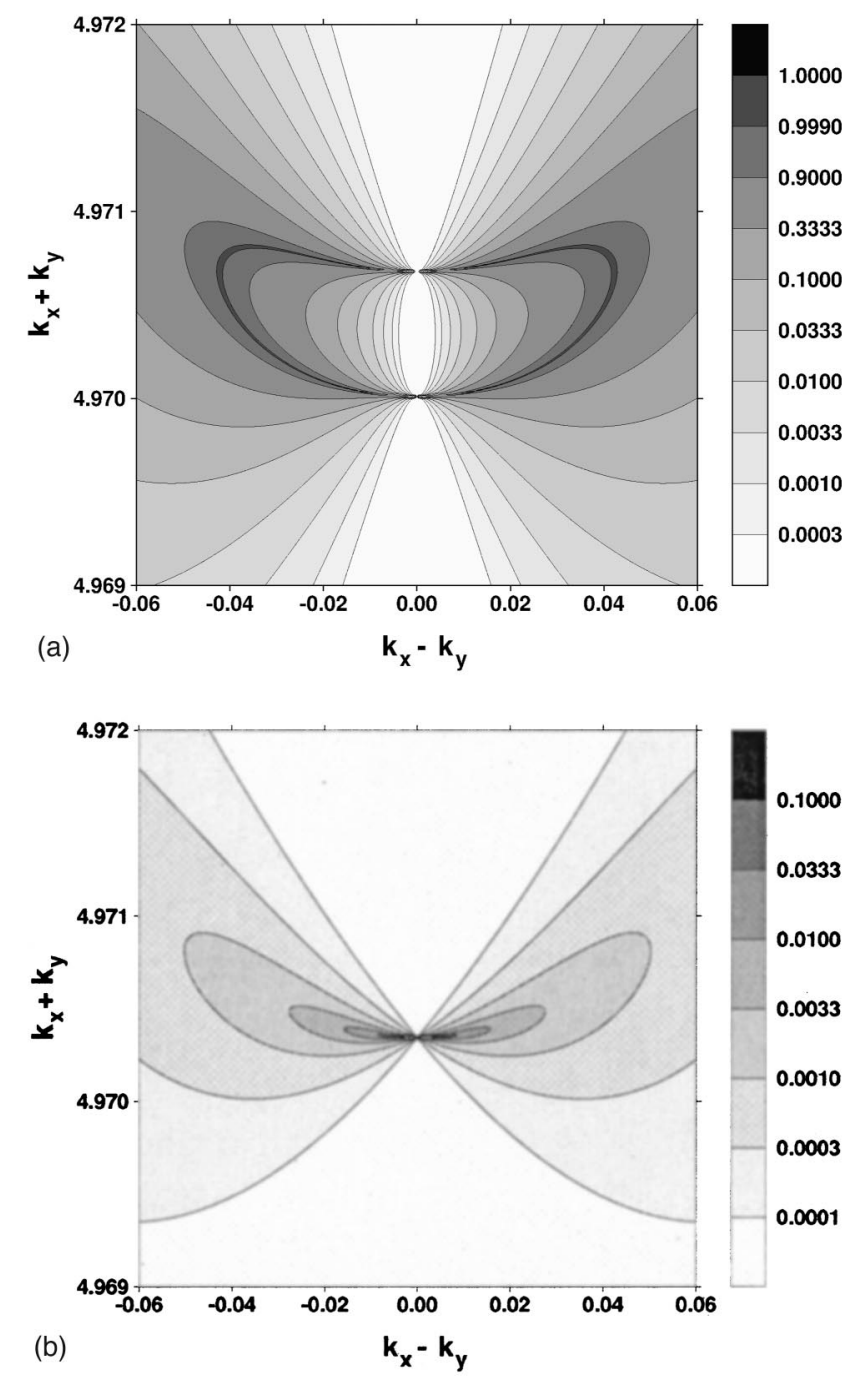

FIG. 5. Enlarged ab initio transmission plot of a Co|3 vac|Co tunnel junctions for (a) parallel aligned moments in the minority band and for (b) antiparallel aligned moments of the Co half spaces. Darker areas correspond to higher transmission probabilities. The reciprocal-lattice vectors are given in units of $1 / a$, where $a$ is the lattice constant of the fcc Co. Note that the intensity scales in (a) and (b) are different by a factor 10 .

ments, in particular the conductance in the minority band, as well as the conductance for the antiparallel alignment.

First we note the important similarities between the above model results and the resonance effects in Fig. 1(b) and Fig. 1(c). In the case of parallel alignment, i.e., for a symmetrical barrier with two degenerate surface states, we find extremely high transmission peaks of nearly 1 , while for the antiparallel alignment where for both spin polarizations only one interface state on one or the other side of the barrier exists, the resonance peaks are reduced by more than two orders of magnitude. This is fully in line with the model results shown in Fig. 2-4.

Let us discuss in more detail the structure of the four dominating double spots in Figs. 1(b) and 1(c), which occur along the $\bar{\Gamma}-\bar{X}$ line, i.e., the diagonal $k_{x}=k_{y}$. The isointen- 
sity contours of the transmission are shown in Figs. 5(a) and 5(b), where darker areas denote higher intensities.

For symmetry reasons the contours show mirror symmetry with respect to the $\bar{\Gamma}-\bar{X}$ line. For the symmetrical case [Fig. 5(a)] all the isointensity lines merge together in two points on this line $k_{x}^{ \pm}=k_{y}^{ \pm}=(2.485172 \pm 0.000167) 1 / a(a$ is the lattice constant of the fcc Co), while for the antiparallel case in Fig. 5(b) all lines merge in a single point at $k_{x}=k_{y}$ $=2.485172) 1 / a$. As indicated by the vanishing linewidth at these points, these are localized states which do not contribute to the current. At these points they coexist with two incident states with full transmission. As the above discussion suggests, in the symmetrical case of Fig. 5(a) the wave functions of the two split localized states are bonding and antibonding combinations of the surface states of the two Co half crystals. In fact in both cases the localization is enforced by symmetry: The localized state is symmetric with respect to a reflection at the $\bar{\Gamma}-\bar{X}$ axis while all propagating states for this $\mathbf{k}_{\|}$point are antisymmetric. However, for a small deviation from the diagonal, other states are intermixed, leading to the loss of orthogonality and to a transition from a localized to a resonant state with a small, but finite halfwidth. Note the large intensity difference between both conductances, which is directly evident even though the intensity scale for the antiparallel case is blown up by a factor 10 . For the parallel alignment the darkest contour area, indicating a transmission larger than 0.999 , contains a line with full transmission 1. With increasing deviation from the diagonal the linewidth increases and the two resonances join together into a single resonance, so that for larger distances the transmission decreases.

For the total conductance one has to integrate the $\mathbf{k}_{\|}$-resolved conductances over the SBZ. It is directly evident from Fig. 5 (Ref. 19) that only the hot spots for parallel alignment will give an important contribution. However the important contribution does not arise from the highest peaks with full transmission, since in Fig. 5(a) these represent only a line, being of measure zero for the integration. The big contributions arise from the whole area around this line, where the transmission is smaller than 1 , but is still strongly enhanced by the partial hopping effect explained above (see Fig. 4). Of course, both effects are directly connected, since this strong enhancement only occurs in the vicinity of the bonding-antibonding resonances. To obtain a more quantitative feeling about the importance of hot spots, we present in Table I the relative areas of the SBZ, for which the transmission lies between 0.1 and 1 , and the relative contributions of these areas to the total conductance for four different junctions with 3 ML of vacuum.

The areas present only a tiny fraction of the SBZ, which makes reliable numerical calculations very complicated. In the case of antiparallel alignment, the $\mathrm{Co} / 3 \mathrm{vac} \mid \mathrm{Co}$ barrier still has hot spots, as seen from Fig. 1(c) or Fig. 5(b), but with the chosen criterion they are of zero importance. The same is true for the stronger asymmetric barrier $\mathrm{Co}|3 \mathrm{vac}| 5 \mathrm{Cu} \mid \mathrm{Co}$ even for the parallel coupling and the minority band. On the other hand for the barrier $\mathrm{Co}|3 \mathrm{vac}| 5 \mathrm{Co} \mid \mathrm{Cu}$, which from a physical point of view is
TABLE I. Contributions to the conductance in the SBZ with a transmission probability between $0.1<T\left(\mathbf{k}_{\|}, E_{F}\right) \leqslant 1$ for different systems (first column). The second column denotes whether the two Co half spaces are aligned antiparallel or parallel, in which case only the spin minority contributions are given. The relative area of the SBZ and the relative contribution to the total conductance are shown in the third and fourth columns.

\begin{tabular}{|c|c|c|c|}
\hline System & & Area $(\%)$ & $\begin{array}{c}\text { Contri- } \\
\text { bution }(\%)\end{array}$ \\
\hline $\mathrm{Co} \mid 3$ vac $\mid \mathrm{Co}$ & antiparallel & 0 & 0 \\
\hline $\mathrm{Co} \mid 3$ vac $\mid \mathrm{Co}$ & minority & 0.0014 & 24.1 \\
\hline $\mathrm{Co}|3 \mathrm{vac}| 5 \mathrm{Co} \mid \mathrm{Cu}$ & minority & 0.0003 & 5.2 \\
\hline $\mathrm{Co} \mid 3 \quad$ vac $|3 \quad \mathrm{Cu}| \mathrm{Co}$ & minority & 0 & 0 \\
\hline $\mathrm{Cu}|5 \mathrm{Co}| 3 \mathrm{vac}|5 \mathrm{Co}| \mathrm{Cu}$ & minority & 0.0471 & 64.9 \\
\hline
\end{tabular}

only slightly asymmetrical, hot spots are still important, even though full transmission cannot occur. Finally for the symmetrical junction $\mathrm{Cu}|5 \mathrm{Co}| 3$ vac| $5 \mathrm{Co} \mid \mathrm{Cu}$ the hot spots are, due to quantum well effects in the Co layers, even more important than for the Co half space junctions.

As our calculations show, high-symmetry lines in the SBZ are favorable for hot spots, since for symmetry reasons localized states are more likely to exist on these lines, so that for nearby $\mathbf{k}_{\|}$values resonance effects will occur. But hot spots can also occur elsewhere in the SBZ.

To our best knowledge, in all calculations hot spots are only observed in the minority band. The most likely reason for this is that the occurrence is limited to a multiband Fermi surface. As our analytical model showed, one needs a second band to provide the coupling of the interface state to the conducting ones, since otherwise the interface state would remain localized.

As we have demonstrated above, large effects from interface resonances in general require symmetrical or nearly symmetrical barriers, since a one-sided resonance is less effective for the tunneling process. It is the formation of bonding and antibonding states that leads to full transmission at the resonance peaks and at the same time to strongly enhanced tunneling due to the partial hopping effect in the vicinity of these resonances. Therefore we believe that interface roughness as well as a finite bias voltage can substantially reduce the importance of hot spots. However, this is a quantitative question since a fully symmetrical barrier is not required and reliable calculations would be highly desirable.

To summarize, we evaluated by model and $a b$ initio calculations the effects of interface states on the conductance of magnetic tunnel junctions. The hot spots found by different groups in $a b$ initio calculations can be explained by the formation of bonding and antibonding hybrids between the interface states on both sides of the barrier. If the resonance condition for these hybrid resonances is met or nearly met, the electron can tunnel through the barrier without or with only little attenuation. The effect explains why the tunneling conductance can be dominated for intermediate barrier thicknesses by interface states, although these hot spots occur only in a tiny fraction of the surface Brillouin zone. Surface states on one side of the interface 
influence the conductance to a smaller degree. Since strong hot spots only occur for a symmetrical or nearly symmetrical barrier, we expect interface roughness and finite bias voltages to reduce the importance of hot spots and interface states in general.

Financial support for this work was provided by the grant agency of the AS CR (Grant No. A1010829), the grant agency of the Czech Republic (Grant No. 202/00/0122), the Ministry of Education of the Czech Republic (Grant No. COST P5.30), the Scientific and Technological Cooperation between Germany and the Czech Republic (Grant No. TSR013-98), the RT Network Computational Magnetoelectronics (Contract No. RTN1-1999-00145), and the TMR-Network Interface Magnetism (Contract No. ERBFMRXCT 960089) of the European Commission.
${ }^{1}$ T. Miyazaki and N. Tezuka, J. Magn. Magn. Mater. 139, L231 (1995).

${ }^{2}$ J.S. Moodera, L.R. Kinder, T.M. Wong, and R. Meservey, Phys. Rev. Lett. 74, 3273 (1995).

${ }^{3}$ J.M. MacLaren, X.-G. Zhang, and W.H. Butler, Phys. Rev. B 56, 11827 (1997).

${ }^{4}$ S. Zhang and P.M. Levy, Phys. Rev. Lett. 81, 5660 (1998).

${ }^{5}$ W.H. Butler, X.-G. Zhang, X. Wang, J. van Ek, and J.M. MacLaren, J. Appl. Phys. 81, 5518 (1997).

${ }^{6}$ I.I. Oleinik, E.Yu. Tsymbal, and D.G. Pettifor, Phys. Rev. B 62, 3952 (2000).

${ }^{7}$ J.M. MacLaren, X.-G. Zhang, W.H. Butler, and Xindong Wang, Phys. Rev. B 59, 5470 (1999).

${ }^{8}$ K. Wang, P. M. Levy, S. Zhang, L. Szunyogh, and P. Weinberger (unpublished).

${ }^{9}$ W.H. Butler, X.-G. Zhang, T.C. Schulthess, and J.M. MacLaren,
Phys. Rev. B 63, 054416 (2001).

${ }^{10}$ J. Mathon and A. Umerski, Phys. Rev. B 63, 220403(R) (2001).

${ }^{11}$ P. Zahn (unpublished).

${ }^{12} \mathrm{Ph}$. Mavropoulos, N. Papanikolaou, and P.H. Dederichs, Phys. Rev. Lett. 85, 1088 (2000).

${ }^{13}$ I.I. Mazin, Europhys. Lett. 55, 404 (2001).

${ }^{14}$ U. Fano, Phys. Rev. 124, 1866 (1961).

${ }^{15}$ P. Bruno, Phys. Rev. B 52, 411 (1995).

${ }^{16}$ K. Wildberger, R. Zeller, P.H. Dederichs, J. Kudrnovský, and P. Weinberger, Phys. Rev. B 58, 13721 (1998).

${ }^{17}$ J. Kudrnovský, V. Drchal, C. Blaas, P. Weinberger, I. Turek, and P. Bruno, Phys. Rev. B 62, 15084 (2000).

${ }^{18}$ T. Kostyrko, Phys. Rev. B 62, 2458 (2000).

${ }^{19}$ The figure was plotted using the software by A. Preusser, ACM Trans. Math. Softw. 15, 79 (1989). 